\title{
Anomalies in the self-diffusion coefficient near the critical point
}

\author{
Binny J. Cherayil \\ Department of Inorganic and Physical Chemistry, Indian Institute of Science, Bangalore-560012, India
}

\begin{abstract}
In this paper we calculate the contribution $D_{s}$ to the self-diffusion coefficient $D_{0}$ from the coupling of solute motion to solvent density fluctuations in an infinitely dilute near-critical binary mixture using the methods of fully renormalized kinetic theory in the disconnected approximation. The expression for $D_{s}$ is found to be similar to mode-coupling expressions for diffusion coefficients in dense or supercooled liquids. As a function of the solvent density $\rho$, and along the critical isotherm, $D_{0}$ exhibits an anomalous decrease in the vicinity of the critical point that originates in the vanishing of $D_{s}$ according to the scaling relation $D_{s} \sim\left(\rho-\rho_{c}\right)^{2}$, where $\rho_{c}$ is the critical density. An analogous decrease in $D_{0}$ has recently been observed in the simulations of Drozdov and Tucker [J. Chem. Phys. 114, 4912 (2001)] on a neat liquid of Lennard-Jones particles, and has been ascribed to local density inhomogeneities in the medium.
\end{abstract}

\section{INTRODUCTION}

Although critical fluctuations are well-known from renormalization group and other calculations to produce anomalies in both static and dynamic quantities, ${ }^{1}$ questions remain about the near-critical behavior of certain transport properties-notably the self-diffusion coefficient $D_{0}$, a parameter implicated in the control of quantum processes in the condensed phase ${ }^{2}$ - for want of reliable experimental data. The customary uncertainties in experimental measurements of critical phenomena appear to be especially pronounced in measurements of diffusion coefficients, which have been found to be anomalous in some experiments, and normal in others. ${ }^{3}$

Theoretical estimates of these coefficients, relying typically on some version of gas-phase theory extended to higher densities, or some version of the Stokes-Einstein theory, modified ad hoc to improve its agreement with experimental data, have not always been in agreement either. ${ }^{4}$ An early mode coupling calculation by Keyes, ${ }^{5}$ for example, suggested that near the critical point, $D_{0}$ shows, at most, a weaker anomaly than the viscosity, while a later calculation by Gitterman ${ }^{6}$ along rather different lines suggested that it vanishes as $\tau^{1.3}, \tau \equiv\left|T-T_{c}\right| / T_{c}$ being the reduced temperature.

Calculations of the mutual diffusivity $D$ in binary mixtures near the critical point have been less ambiguous: they suggest that $D$ vanishes like the inverse correlation length $\xi$ along the critical isochore. ${ }^{7}$ Since $D$ reduces to $D_{0}$ in the limit of vanishing amounts of one component, these results seem to imply that $D_{0}$ should also decrease anomalously. Evidence for such behavior has been seen in recent simulations by Drozdov and Tucker ${ }^{8}$ on a neat Lennard-Jones fluid. The anomaly here has been ascribed to critical point changes in the density profile of the fluid surrounding the tagged particle, and is in a direction opposite to anomalies in normal dense mixtures of solutes in solvents of larger size, which show an enhancement over the Stokes-Einstein prediction. ${ }^{9}$
The present paper is an attempt to provide an independent theoretical estimate of $D_{0}$ in dilute near-critical binary mixtures using the fully renormalized kinetic theory (FRKT) developed by Mazenko and co-workers. ${ }^{10-17}$ The FRKT formalism, in addition to providing a first principles phase space approach to particle dynamics, is naturally suited to a description of the near-critical region, as it systematically replaces bare by renormalized interactions, thereby ensuring the development of an effectively long-ranged interparticle potential on approach to the critical point. This renormalized potential allows for the introduction of well-controlled approximations into the dynamical equations governing selfmotion. The FRKT formalism therefore offers an alternative to approaches based on mode coupling theories. The present calculations confirm the existence of an anomalous decrease in $D_{0}$ near the critical point, and suggest that it is primarily the coupling of solute motion to solvent density fluctuations that is the cause.

The following section briefly sets down the arguments for identifying the density fluctuations of the solvent as the principal component of $D_{0}$ in the neighborhood of the critical point. The FRKT formalism is then introduced and developed in Sec. III to treat this component. Although FRKT methods have been extensively reviewed, they are nevertheless discussed here in some detail in the interests of completeness, and in order to provide a single unifying perspective. The expressions derived in this section are largely exact, but opaque, so a number simplifying approximations are introduced in Secs. IV and V to obtain more transparent analytic results, which are then used in the concluding section to derive scaling relations for the diffusion coefficient, to estimate its actual numerical values under different thermodynamic conditions for a chosen model system, and to compare the general results with mode-coupling and other approaches. 


\section{CONTRIBUTIONS TO SELF-MOTION}

Three important dynamical processes contribute to the motion of a solute particle through a solvent: direct binary collisions, collective solvent density fluctuations, and transverse solvent currents. ${ }^{9,18}$ (Longitudinal current effects can also contribute, but they are generally negligible.) If the solvent is dense, the inertial component of the motion, associated with pairwise interactions between individual molecules, is usually—but not always-small; $;{ }^{9,18}$ as a first approximation, therefore, it is omitted in these calculations, which are interested primarily in identifying some minimal set of processes that could plausibly account for an anomalous decrease in $D_{0}$. If attention is further restricted at this stage to the asymptotic near-critical region, where the decay of the density fluctuations is slow, the contributions from transverse solvent currents can likewise be ignored. The approximation should probably be avoided in a more sophisticated formulation of the theory, since fluids near the critical point are not necessarily as dense or as slow as the glassy or supercooled liquids to which the approximation is most likely to apply. This leaves the collective density fluctuations of the solvent, which act to "cage" the motion of the solute, as the dominant effect controlling the behavior of $D_{0}$. Near the critical point of the solvent, therefore, it is assumed that

$$
D_{0} \approx D_{s},
$$

where $D_{s}$ is identified with self-motion governed solely by solvent density fluctuations in the immediate neighborhood of the critical point. The anomalies in $D_{0}$ are therefore assumed to originate wholly from the anomalies-if any-in the behavior of $D_{s}$. The basic goal of the paper is therefore to derive an expression for $D_{s}$, and this is done using the methods of renormalized kinetic theory. The calculations are quite involved, so readers uninterested in the mathematical details can skip the next several sections and proceed directly to Eq. (56), which is the final expression for $D_{s}$.

\section{KINETIC THEORY}

\section{A. Definitions}

The dynamical state of a mixture of $N_{1}$ particles of the solute (component 1) and $N_{2}$ particles of the solvent (component 2) is characterized completely by their positions and momenta, which (for self-motion) are specified by the following phase space densities:

$$
\begin{aligned}
& f_{s}^{(1)}(1)=\sqrt{N} \delta\left(\mathbf{r}_{1}-\mathbf{R}_{1}^{(1)}\right) \delta\left(\mathbf{p}_{1}-\mathbf{P}_{1}^{(1)}\right), \\
& f^{(2)}(1)=\sum_{i=1}^{N_{2}} \delta\left(\mathbf{r}_{1}-\mathbf{R}_{i}^{(2)}\right) \delta\left(\mathbf{p}_{1}-\mathbf{P}_{i}^{(2)}\right) .
\end{aligned}
$$

Here $N=N_{1}+N_{2}$, while $\mathbf{R}_{i}^{(\alpha)}$ and $\mathbf{P}_{i}^{(\alpha)}$ are the position and momentum of the $i$ th particle of the $\alpha$ th component, whose actual values, enforced by the delta functions, are given by the field variables $\mathbf{r}_{n}$ and $\mathbf{p}_{n}$. Following Mazenko, ${ }^{15}$ we use the abbreviation $n \equiv \mathbf{r}_{n}, \mathbf{p}_{n}$ to refer to these variables. The subscript $s$ distinguishes self from collective phase space densities. (Recall that $D_{0}$ is the self-diffusion coefficient at any given temperature and density; $D_{s}$ is the self-diffusion coefficient in the vicinity of the critical point under condi- tions where solvent density fluctuations are assumed to be dominant.) To determine $D_{s}$ for a tagged particle of the solute (component 1), we require the following dynamical correlation function ${ }^{15}$

$$
C_{s}^{(11)}(12 ; z)=\left\langle f_{s}^{(1)}(2)(z+L)^{-1} f_{s}^{(1)}(1)\right\rangle,
$$

where $z$ is a complex frequency (the Laplace conjugate of the time $t$ ), and $L$ is the Liouville operator, defined by

$$
L=L_{0}^{(1)}+L_{0}^{(2)}+L_{I}^{(11)}+L_{I}^{(22)}+L_{I}^{(12)},
$$

where

$$
\begin{aligned}
& L_{0}^{(\alpha)}=-\frac{i}{m_{\alpha}} \sum_{i=1}^{N_{\alpha}} \mathbf{P}_{i}^{(\alpha)} \cdot \nabla_{\mathbf{R}_{i}^{(\alpha)}}, \\
& L_{I}^{(\alpha \alpha)}=i \sum_{i=1}^{N_{\alpha}} \sum_{j \neq i}^{N_{\alpha}} \nabla_{\mathbf{R}_{i}^{(\alpha)}} V^{(\alpha \alpha)}\left(\mathbf{R}_{i}^{(\alpha)}-\mathbf{R}_{j}^{(\alpha)}\right) \cdot \nabla_{\mathbf{P}_{i}^{(\alpha)},} \\
& L_{I}^{(12)}=i \sum_{i=1}^{N_{1}} \sum_{j=1}^{N_{2}} \nabla_{\mathbf{R}_{i}^{(1)}} V^{(12)}\left(\mathbf{R}_{i}^{(1)}-\mathbf{R}_{j}^{(2)}\right) \cdot\left(\nabla_{\mathbf{P}_{i}^{(1)}}-\nabla_{\left.\mathbf{P}_{j}^{(2)}\right)} .\right.
\end{aligned}
$$

$V^{(11)}, V^{(22)}$ and $V^{(12)}$ are the solute-solute, solvent-solvent and solute-solvent pair potentials, respectively, while $m_{\alpha}$ is the mass of component $\alpha$. In Eq. (4), the angular brackets denote an equilibrium ensemble average over the initial phase space distribution function of the mixture, so $C_{s}^{(11)}(12 ; z)$ is the Laplace transform of an equilibrium phase space time correlation function. The superscript (11) [and in general $(\alpha \alpha)$ ] identifies the correlation function as an autocorrelation function. [Later expressions will use the superscripts (12) or (21) - these will refer to distinct correlation functions.]

\section{B. Equation-of-motion for $C_{s}^{(11)}(12 ; z)$}

The operator identity $(A+B)^{-1}=A^{-1}-A^{-1}(A$ $+B)^{-1} B$ may be used to write the correlation function as

$$
\begin{aligned}
C_{s}^{(11)}(12 ; z)= & \frac{1}{z} \widetilde{C}_{s}^{(12)}(12)-\frac{1}{z}\left\langle f_{s}^{(1)}(2)(z\right. \\
& \left.+L)^{-1} L f_{s}^{(1)}(1)\right\rangle,
\end{aligned}
$$

where the tilde denotes the equilibrium value of the correlation function (which is assumed to be known exactly). Using properties of the delta function (such as, $\partial \delta(x-y) / \partial x$ $=-\partial \delta(x-y) / \partial y)$, the action of the Liouville operator on the phase space density may now be re-expressed in terms operators involving only the field variables. In this way, it is easily shown that

$$
\begin{aligned}
& L_{0}^{(1)} f_{s}^{(1)}(1)=-L_{0}^{(1)}(1) f_{s}^{(1)}(1), \\
& L_{0}^{(2)} f_{s}^{(1)}(1)=0, \\
& L_{I}^{(11)} f_{s}^{(1)}(1)=-\int d \overline{1} L_{I}^{(11)}(1 \overline{1}) g^{(11)}(1 \overline{1}), \\
& L_{I}^{(22)} f_{s}^{(1)}(1)=0, \\
& L_{I}^{(12)} f_{s}^{(1)}(1)=-\int d \overline{1} L_{I}^{(12)}(1 \overline{1}) g^{(12)}(1 \overline{1}),
\end{aligned}
$$


where

$$
\begin{aligned}
& L_{0}^{(1)}(1)=-\frac{i}{m_{1}} \mathbf{p}_{1} \cdot \nabla_{\mathbf{r}_{1}}, \\
& L_{I}^{(11)}(1 \overline{1})=i \nabla_{\mathbf{r}_{1}} V^{(11)}\left(\mathbf{r}_{1}-\mathbf{r}_{1}^{-}\right) \cdot\left(\nabla_{\mathbf{p}_{1}}-\nabla_{\mathbf{p}_{1}^{-}}\right), \\
& L_{I}^{(12)}(1 \overline{1})=i \nabla_{\mathbf{r}_{1}} V^{(12)}\left(\mathbf{r}_{1}-\mathbf{r}_{1}^{-}\right) \cdot\left(\nabla_{\mathbf{p}_{1}}-\nabla_{\mathbf{p}_{1}^{-}}\right),
\end{aligned}
$$

with the higher-order correlation functions $g^{(11)}(1 \overline{1})$ and $g^{(12)}(1 \overline{1})$ being defined as $g^{(11)}(1 \overline{1})=f_{s}^{(1)}(1) \Sigma_{i \neq 1}^{N_{1}} \delta\left(\mathbf{r}_{1}^{-}\right.$ $\left.-\mathbf{R}_{i}^{(1)}\right) \delta\left(\mathbf{p}_{1}^{-}-\mathbf{P}_{i}^{(1)}\right) \quad$ and $\quad g^{(12)}(1 \overline{1})=f_{s}^{(1)}(1) f^{(2)}(\overline{1})$. The symbol $\int d n$ denotes an integration over the variables $\mathbf{r}_{n}$ and $\mathbf{p}_{n}$. When Eqs. (10)-(14) are substituted into Eq. (9), we obtain a dynamical equation for $C_{s}^{(11)}(12 ; z)$ :

$$
\begin{aligned}
{[z-} & \left.L_{0}^{(1)}(1)\right] C_{s}^{(11)}(12 ; z) \\
= & \widetilde{C}_{s}^{(11)}(12)+\int d \overline{1} L_{I}^{(11)}(1 \overline{1})\left\langle f_{s}^{(1)}(2)(z+L)^{-1}\right. \\
& \left.\times g^{(11)}(1 \overline{1})\right\rangle+\int d \overline{1} L_{I}^{(12)}(1 \overline{1})\left\langle f_{s}^{(1)}(2)(z+L)^{-1}\right. \\
& \left.\times g^{(12)}(1 \overline{1})\right\rangle \\
\equiv & \widetilde{C}_{s}^{(11)}(12)+\int d \overline{1} \phi_{s}^{(11)}(1 \overline{1} ; z) C_{s}^{(11)}(\overline{1} 2 ; z) .
\end{aligned}
$$

The second equality in the above expressions is the definition of a new dynamical correlation function $\phi_{s}^{(11)}(1 \overline{1} ; z)$. The terms on the left-hand side of Eq. (18) describe "free streaming" motion (motion that occurs in the absence of intermolecular forces), so the correlation function defined by Eq. (19) — called a memory function - contains, by construction, all of the remaining dynamical many body effects that appear when intermolecular forces are present.

\section{The memory function}

From Eq. (19), the definition of the memory function is seen to be

$$
\begin{aligned}
& \int d \overline{1} \phi_{s}^{(11)}(1 \overline{1} ; z) C_{s}^{(11)}(\overline{1} 2 ; z) \\
& =\int d \overline{1} L_{I}^{(11)}(1 \overline{1})\left\langle f_{s}^{(1)}(2)(z+L)^{-1} g^{(11)}(1 \overline{1})\right\rangle \\
& \quad+\int d \overline{1} L_{I}^{(12)}(1 \overline{1})\left\langle f_{s}^{(1)}(2)(z+L)^{-1} g^{(12)}(1 \overline{1})\right\rangle .
\end{aligned}
$$

An exact but formal expression for the memory function is now obtained as follows: Eq. (20) is multiplied by $C_{s}^{(11)}(2 \overline{2} ; z)^{-1}$ and integrated over 2 (i.e., over $\mathbf{r}_{2}$ and $\mathbf{p}_{2}$ ) to produce: $\phi_{s}^{(11)}(1 \overline{1} ; z)$

$$
\begin{aligned}
= & \int d \overline{3} d \overline{4} L_{I}^{(11)}(1 \overline{3}) C_{s}^{(111)}(1 \overline{3} ; \overline{4} ; z) C_{s}^{(11)}(\overline{4} \overline{1} ; z)^{-1} \\
& +\int d \overline{3} d \overline{4} L_{I}^{(12)}(1 \overline{3}) C_{s}^{(121)}(1 \overline{3} ; \overline{4} ; z) C_{s}^{(11)}(\overline{4} \overline{1} ; z)^{-1},
\end{aligned}
$$

where

$$
C_{s}^{(111)}(1 \overline{3} ; \overline{4} ; z) \equiv\left\langle f_{s}^{(1)}(\overline{4})(z+L)^{-1} g^{(11)}(1 \overline{3})\right\rangle
$$

and

$$
C_{s}^{(121)}(1 \overline{3} ; \overline{4} ; z) \equiv\left\langle f_{s}^{(1)}(\overline{4})(z+L)^{-1} g^{(12)}(1 \overline{3})\right\rangle .
$$

If we introduce an adjoint form of Eq. (4), defined as

$$
C_{s}^{(11)}(12 ; z)=\left\langle f_{s}^{(1)}(1)(z-L)^{-1} f_{s}^{(1)}(2)\right\rangle,
$$

an equation-of-motion analogous to Eq. (18) is easily derived; it is given by

$$
\begin{aligned}
{[z+} & \left.L_{0}^{(1)}(2)\right] C_{s}^{(11)}(12 ; z) \\
= & \widetilde{C}_{s}^{(11)}(12)-\int d \overline{2} L_{I}^{(11)}(2 \overline{2}) \\
& \times\left\langle f_{s}^{(1)}(1)(z-L)^{-1} g^{(11)}(2 \overline{2})\right\rangle \\
& -\int d \overline{2} L_{I}^{(12)}(2 \overline{2})\left\langle f_{s}^{(1)}(1)(z-L)^{-1} g^{(12)}(2 \overline{2})\right\rangle .
\end{aligned}
$$

The operator $\left[z+L_{0}^{(1)}(2)\right]$ is now applied to both sides of Eq. (20), combined with Eq. (25) and simplified. Equation (21) is then substituted into the result. After relabeling, it can be shown that ${ }^{19}$

$$
\begin{aligned}
\int d & \phi_{s}^{(11)}(1 \overline{1}) \widetilde{C}_{s}^{(11)}(\overline{1} 2) \\
= & -\sum_{\alpha=1}^{2} \sum_{\beta=1}^{2} \int d \overline{1} d \overline{2} L_{I}^{(1 \alpha)}(1 \overline{1}) \\
& \left.\quad \times L_{I}^{(1 \beta)}(2 \overline{2}) G_{s}^{(1 \alpha 1 \beta}\right)(1 \overline{1} ; 2 \overline{2} ; z),
\end{aligned}
$$

where $G_{s}^{(1 \alpha 1 \beta)}(1 \overline{1} ; 2 \overline{2} ; z)$ is a dynamical four-point correlation function that is defined as

$$
\begin{aligned}
G_{s}^{(1 \alpha 1 \beta)} & (1 \overline{1} ; 2 \overline{2} ; z) \\
= & C_{s}^{(1 \alpha 1 \beta)}(1 \overline{1} ; 2 \overline{2} ; z)-\int d \overline{3} d \overline{4} C_{s}^{(1 \alpha 1)}(1 \overline{1} ; \overline{3} ; z) \\
& \times C_{s}^{(11)}(\overline{3} \overline{4} ; z)^{-1} C_{s}^{(11 \beta)}(\overline{4} ; 2 \overline{2} ; z) .
\end{aligned}
$$

(The three- and four-point correlation functions of Eqs. (22) -(27) are identified by superscripts that match the superscripts of the phase space densities that appear in their definitions. For instance, in Eq. (23), the first two indices of the superscript (121) refer to the phase space density that follows the Liouville operator, while the last index refers to the phase space density that precedes the operator. The arguments of the three-point correlation function are just the arguments of the corresponding phase space densities.) 


\section{Vertex interactions}

It proves useful to introduce a new four-point correlation function $\bar{G}_{s}^{(\alpha \beta \gamma \delta)}(a b ; c d ; z)$, defined implicitly by ${ }^{15}$

$$
\begin{aligned}
G_{s}^{(1 \alpha 1 \beta)}(1 \overline{1} ; 2 \overline{2} ; z) \equiv & \sum_{\mu=1}^{2} \sum_{\nu=1}^{2} \int d 3 d \overline{3} d 4 d \overline{4} \\
& \times \widetilde{G}_{s}^{(1 \alpha 1 \mu)}(1 \overline{1} ; 3 \overline{3}) \bar{G}_{s}^{(1 \mu 1 \nu)}(3 \overline{3} ; 4 \overline{4} ; z) \\
& \times \widetilde{G}_{s}^{(1 \nu 1 \beta)}(4 \overline{4} ; 2 \overline{2})
\end{aligned}
$$

where the tilde denotes, as before, the equilibrium value of the correlation function. Using Eq. (28) in Eq. (26), the memory function can now be written as

$$
\begin{aligned}
& \int d \overline{1} \phi_{s}^{(11)}(1 \overline{1} ; z) \widetilde{C}_{s}^{(11)}(\overline{1} 2) \\
& =-\sum_{\mu=1}^{2} \sum_{\nu=1}^{2} \int d 3 d \overline{3} d 4 d \overline{4} \mathcal{V}^{(11 \mu)}(1 ; 3 \overline{3}) \\
& \quad \times \bar{G}_{s}^{(1 \mu 1 \nu)}(3 \overline{3} ; 4 \overline{4} ; z) \mathcal{V}^{(11 \nu)}(2 ; 4 \overline{4}),
\end{aligned}
$$

where the vertex function $\mathcal{V}^{(11 \mu)}(1 ; 3 \overline{3})$ is given by

$$
\mathcal{V}^{(11 \mu)}(1 ; 3 \overline{3}) \equiv \sum_{\alpha=1}^{2} \int d \overline{1} L_{I}^{(1 \alpha)}(1 \overline{1}) \widetilde{G}_{s}^{(1 \alpha 1 \mu)}(1 \overline{1} ; 3 \overline{3}) .
$$

A similar definition holds for the vertex function $\mathcal{V}^{(11 \nu)}(2 ; 4 \overline{4})$. These functions are purely static quantities, i.e., they do not depend on the frequency $z$. They can be computed exactly in terms of the familiar pair correlation functions of equilibrium statistical mechanics when triplet correlations between particles are ignored. Under these conditions ${ }^{12,15}$

$$
\widetilde{G}_{s}^{(1 \alpha 1 \mu)}(1 \overline{1} ; 3 \overline{3})=\widetilde{C}_{s}^{(1 \alpha 1 \mu)}(1 \overline{1} ; 3 \overline{3}),
$$

where

$$
\widetilde{C}_{s}^{(1 \alpha 1 \mu)}(1 \overline{1} ; 3 \overline{3})=\left\langle g^{(1 \mu)}(3 \overline{3})(z+L)^{-1} g^{(1 \alpha)}(1 \overline{1})\right\rangle .
$$

Substituting Eqs. (31) and (32) into the definition of the vertex function, and using the properties of the Liouville operator in conjunction with the definition of the equilibrium average, one can derive the following relations:

$$
\mathcal{V}^{(\alpha \alpha \mu)}=-\omega_{o}^{(\alpha \mu)} \tilde{L}_{I}^{(\alpha \mu)}(3 \overline{3}) \delta(1 \overline{3}),
$$

where

$$
\omega_{o}^{(\alpha \mu)}=\rho_{\alpha} \rho_{\mu} f_{o}^{(\alpha)}\left(\mathbf{p}_{3}\right) f_{o}^{(\mu)}\left(\mathbf{p}_{\overline{3}}^{-}\right) g^{(\alpha \mu)}\left(\mathbf{r}_{3}-\mathbf{r}_{3}^{-}\right)
$$

and

$$
\widetilde{L}_{I}^{(\alpha \mu)}=-\frac{i}{\beta} \nabla_{\mathbf{r}_{3}} \ln g^{(\alpha \mu)}\left(\mathbf{r}_{3}-\mathbf{r}_{3}^{-}\right) \cdot\left(\nabla_{\mathbf{p}_{3}}-\nabla_{\mathbf{p}_{3}^{-}}\right) .
$$

Here, $\beta=1 / k_{B} T, \rho_{\alpha}$ is the number density of the $\alpha$ th component, $f_{o}^{(\alpha)}(\mathbf{p})$ is its normalized Boltzmann velocity distribution, $g^{(\alpha \mu)}\left(\mathbf{r}-\mathbf{r}^{\prime}\right)$ is the radial distribution function between the two components and $\delta(13) \equiv \delta\left(\mathbf{r}_{1}-\mathbf{r}_{3}\right) \delta\left(\mathbf{p}_{1}\right.$ $-\mathbf{p}_{3}$ ). The vertex function is thus seen to be related to the potential of mean force [which is proportional to $\ln g^{(\alpha \mu)}\left(\mathbf{r}_{3}\right.$ $\left.-\mathbf{r}_{3}^{-}\right)$], the original bare interactions having been replaced by fully renormalized interactions.

\section{APPROXIMATIONS}

One could extend the above formalism further by expressing the four-point correlation function in terms of still higher-order correlation functions, but the usefulness of this procedure is doubtful. The strategy at this stage is therefore to use physical considerations to arrive at reasonable approximations to the existing correlation functions. ${ }^{15,16,19}$ The first step in this strategy is to invert the equation defining $\bar{G}_{s}$ [Eq. (28)]. In real time $t$ the result is

$$
\begin{aligned}
\bar{G}_{s}^{(1 \mu 1 \nu)}(3 \overline{3} ; 4 \overline{4} ; t) & \\
= & \sum_{\alpha, \beta=1}^{2} \int d 1 d \overline{1} d 2 d \overline{2} \widetilde{G}_{s}^{(1 \alpha 1 \mu)}(3 \overline{3} ; 1 \overline{1})^{-1} \\
& \times G_{s}^{(1 \alpha 1 \beta)}(1 \overline{1} ; 2 \overline{2} ; t) \widetilde{G}_{s}^{(1 \nu 1 \beta)}(2 \overline{2} ; 4 \overline{4} ; t)^{-1},
\end{aligned}
$$

which, when combined with Eq. (29), leads to

$$
\begin{aligned}
\phi_{s}^{(11)}(12 ; t) \rho_{1} f_{o}^{(1)}\left(\mathbf{p}_{2}\right) \\
=-\sum_{\alpha, \beta=1}^{2} \sum_{\mu, \nu=1}^{2} \int d 3 d \overline{3} d 4 d \overline{4} d 5 d \overline{5} d 6 d \overline{6} \\
\quad \times \mathcal{V}^{(11 \mu)}(1 ; 3 \overline{3}) \widetilde{G}_{s}^{(1 \alpha 1 \mu)}(3 \overline{3} ; 5 \overline{5})^{-1} G_{s}^{(1 \alpha 1 \beta)}(5 \overline{5} ; 6 \overline{6} ; t) \\
\quad \times \widetilde{G}_{s}^{(1 \nu 1 \beta)}(6 \overline{6} ; 4 \overline{4})^{-1} \mathcal{V}^{(11 \nu)}(2 ; 4 \overline{4}) .
\end{aligned}
$$

When component 2 (the solvent) is near its critical point, the expression for the memory function can be simplified by virtue of the so-called disconnected approximation ${ }^{14,15,19}$ for the four-point correlation function $G_{s}^{(\alpha \beta \gamma \delta)}(a b ; c d ; z)$. In general, this function can be written exactly as the sum of a connected contribution and a disconnected contribution. The connected part of the function vanishes when its field points are separated into sets that are spatially independent; under the same operation, the disconnected part remains finite. ${ }^{19}$ Near the critical point, the physical processes that are important in determining $\bar{G}_{s}$ are expected to be governed by the collective effects associated with the onset of long-range order. Such effects involve pairs of particles that first collide, then move apart under the influence of the medium but independently of each other, and then eventually recollide. The process is collective in that the correlations that lead to recollision are mediated by the environment. It is reasonable, therefore, to approximate $G_{s}^{(\alpha \beta \gamma \delta)}(a b ; c d ; z)$ by its disconnected part. ${ }^{14}$ This is helpful because under this approximation we have the exact relation

$$
G_{s}^{(\alpha \mu \alpha \nu)}(1 \overline{1} ; 2 \overline{2} ; t)=C_{s}^{(\alpha \alpha)}(12 ; t) C^{(\mu \nu)}(\overline{1} \overline{2} ; t),
$$

which also holds for the equilibrium value of the correlation function. Now it is easily shown that

$$
C_{s}^{(\alpha \alpha)}(12)=\rho_{\alpha} f_{o}^{(\alpha)}\left(\mathbf{p}_{1}\right) \delta(12)
$$

and 


$$
\begin{aligned}
\widetilde{C}_{s}^{(\alpha \beta)}(12)= & \delta_{\alpha \beta} \delta(12) \rho_{\alpha} f_{o}^{(\alpha)}\left(\mathbf{p}_{1}\right) \\
& +\rho_{\alpha} \rho_{\beta} f_{o}^{(\alpha)}\left(\mathbf{p}_{1}\right) f_{o}^{(\beta)}\left(\mathbf{p}_{2}\right) h^{(\alpha \beta)}\left(\mathbf{r}_{1}-\mathbf{r}_{2}\right),
\end{aligned}
$$

where $h^{(\alpha \beta)}\left(\mathbf{r}_{1}-\mathbf{r}_{2}\right)$ is the indirect correlation function introduced by Ornstein and Zernike. ${ }^{20}$ Hence,

$$
\widetilde{C}_{s}^{(\alpha \alpha)}(12)^{-1}=\delta(12) / \omega_{o}^{(\alpha)}(1),
$$

where $\omega_{o}^{(\alpha)}(1)=\rho_{\alpha} f_{o}^{(\alpha)}\left(\mathbf{p}_{1}\right)$, and

$$
\widetilde{C}^{(\alpha \beta)}(12)^{-1}=\delta_{\alpha \beta} \delta(12) / \omega_{o}^{(\alpha)}(1)-c^{(\alpha \beta)}\left(\mathbf{r}_{1}-\mathbf{r}_{2}\right),
$$

with $c^{(\alpha \beta)}\left(\mathbf{r}_{1}-\mathbf{r}_{2}\right)$ being the direct correlation function between the two components. These results, when substituted into Eq. (37), produce, after lengthy algebra, the following expression for the memory function in Fourier space:

$$
\begin{aligned}
\phi_{s}^{(11)}\left(\mathbf{k} ; \mathbf{p}_{1}, \mathbf{p}_{2} ; t\right) & \\
= & \frac{1}{\omega_{o}^{(1)}(2) \beta^{2}} \sum_{\alpha, \beta}^{2} \int d \mathbf{q q} \cdot \nabla_{\mathbf{p}_{1}} \mathbf{q} \cdot \nabla_{\mathbf{p}_{2}} S^{(\alpha \beta)}(\mathbf{q}, t) \\
& \times C_{s}^{(11)}\left(\mathbf{k}-\mathbf{q} ; \mathbf{p}_{1}, \mathbf{p}_{2} ; t\right) c^{(1 \alpha)}(\mathbf{q}) c^{(1 \beta)}(-\mathbf{q}),
\end{aligned}
$$

where

$$
S^{(\alpha \beta)}(\mathbf{q}, t)=\int d \mathbf{p}_{1} d \mathbf{p}_{2} C^{(\alpha \beta)}\left(\mathbf{q} ; \mathbf{p}_{1}, \mathbf{p}_{2} ; t\right)
$$

is the definition of the dynamical structure factor of the mixture.

When one of the components of the mixture (say, 1) is infinitely dilute, Eq. (43) may be simplified further to

$$
\begin{aligned}
\phi_{s}^{(11)}\left(\mathbf{k} ; \mathbf{p}_{1}, \mathbf{p}_{2} ; t\right) \\
=\frac{1}{\omega_{o}^{(1)}(2) \beta^{2}} \int d \mathbf{q q} \cdot \nabla_{\mathbf{p}_{1}} \mathbf{q} \cdot \nabla_{\mathbf{p}_{2}} S^{(22)}(\mathbf{q}, t) \\
\quad \times C_{s}^{(11)}\left(\mathbf{k}-\mathbf{q} ; \mathbf{p}_{1}, \mathbf{p}_{2} ; t\right)\left|c^{(12)}(\mathbf{q})\right|^{2},
\end{aligned}
$$

which is a generalization of the result derived by Mazenko for the memory function of a one-component plasma. ${ }^{14}$

\section{FURTHER APPROXIMATIONS}

In general, $D_{s}$ may be obtained from ${ }^{15}$

$$
D_{s}=\lim _{z \rightarrow i 0^{+}} i V(z)
$$

where

$$
V(z)=-\frac{i}{3} \int_{0}^{\infty} d t \mathrm{e}^{i z t}\left\langle\mathbf{v}_{1}(t) \cdot \mathbf{v}_{1}(0)\right\rangle
$$

with $\mathbf{v}_{1}(t)$ the velocity at time $t$ of the tagged particle. The following identity relates $V(z)$ to the two-point correlation function of the tagged particle introduced earlier:

$$
V(z)=\lim _{\mathbf{k} \rightarrow 0} \frac{1}{3 \rho_{1} m_{1}^{2}} \int d \mathbf{p}_{1} d \mathbf{p}_{2} \mathbf{p}_{1} \cdot \mathbf{p}_{2} C_{s}^{(11)}\left(\mathbf{k} ; \mathbf{p}_{1}, \mathbf{p}_{2} ; z\right) .
$$

The two-point correlation function in turn can be expressed in terms of the memory function through

$$
\begin{aligned}
(z- & \left.\frac{1}{m_{1}} \mathbf{p}_{1} \cdot \mathbf{k}\right) C_{s}^{(11)}\left(\mathbf{k} ; \mathbf{p}_{1}, \mathbf{p}_{2} ; z\right) \\
& -\int d \mathbf{p}_{1}^{-} \phi_{s}^{(11)}\left(\mathbf{k} ; \mathbf{p}_{1}, \mathbf{p}_{1}^{-} ; z\right) C_{s}^{(11)}\left(\mathbf{k} ; \mathbf{p}_{1}^{-}, \mathbf{p}_{2} ; z\right) \\
& =\widetilde{C}_{s}^{(11)}\left(\mathbf{k} ; \mathbf{p}_{1}, \mathbf{p}_{2}\right) .
\end{aligned}
$$

The above equation for $C_{s}^{(11)}\left(\mathbf{k} ; \mathbf{p}_{1}, \mathbf{p}_{2} ; z\right)$ can be solved in principle, given the expression for the memory function defined by Eq. (45). The procedure involves the expansion of the phase space correlation functions $C_{s}^{(11)}\left(\mathbf{k} ; \mathbf{p}_{1}, \mathbf{p}_{2} ; t\right)$ and $C^{(22)}\left(\mathbf{q} ; \mathbf{p}_{1}, \mathbf{p}_{2} ; t\right)$ in a complete orthonormal set of momentum eigenfunctions representing hydrodynamic and nonhydrodynamic modes of excitation. ${ }^{15}$ (The hydrodynamic modes are governed by the slow variables that are associated with the conservation of mass, momentum, and energy.) Rather than pursue this approach, however, which is technically challenging, we simply assume that the behavior is dominated by the single hydrodynamic mode associated with number conservation, so that

$$
C_{s}^{(11)}\left(\mathbf{k} ; \mathbf{p}_{1}, \mathbf{p}_{2} ; t\right)=f_{o}\left(\mathbf{p}_{1}\right) f_{o}\left(\mathbf{p}_{2}\right) S_{s}^{(11)}(\mathbf{k}, t),
$$

where

$$
S_{s}^{(11)}(\mathbf{k}, t)=\int d \mathbf{p}_{1} d \mathbf{p}_{2} C_{s}^{(11)}\left(\mathbf{k} ; \mathbf{p}_{1}, \mathbf{p}_{2} ; t\right) .
$$

Now in the $\mathbf{k}=0$ limit, Eq. (49) can be inverted as

$$
\begin{aligned}
C_{s}^{(11)}\left(\mathbf{0} ; \mathbf{p}_{1}, \mathbf{p}_{2} ; z\right)= & \int d \mathbf{p}_{1}^{-}\left[z \delta\left(\mathbf{p}_{1}-\mathbf{p}_{1}^{-}\right)\right. \\
& \left.-\phi_{s}^{(11)}\left(\mathbf{0} ; \mathbf{p}_{1}, \mathbf{p}_{1}^{-} ; z\right)\right]^{-1} \widetilde{C}_{s}^{(11)}\left(\mathbf{0} ; \mathbf{p}_{1}^{-}, \mathbf{p}_{2}\right) .
\end{aligned}
$$

To a good approximation, it can be assumed that the memory function is diagonal in the momentum eigenfunctions. ${ }^{15,19}$ Equation (48) for $V(z)$ may then be approximated by

$$
V(z) \approx \frac{k_{B} T}{m_{1}} \frac{1}{z-R(z) / 3 m_{1} k_{B} T},
$$

where

$$
R(z)=\int d \mathbf{p}_{1} d \mathbf{p}_{2} \mathbf{p}_{1} \cdot \mathbf{p}_{2} \phi_{s}^{(11)}\left(\mathbf{0} ; \mathbf{p}_{1}, \mathbf{p}_{2} ; z\right) f_{o}^{(1)}\left(\mathbf{p}_{2}\right),
$$

which, using Eqs. (45) and (50), produces

$$
\begin{aligned}
R(z)= & -i \frac{\left(k_{B} T\right)^{2}}{\rho_{1}} \int_{0}^{\infty} d t \int d \mathbf{q}^{i z t} \mathbf{q}^{2} S_{s}^{(11)}(\mathbf{q}, t) \\
& \times S^{(22)}(\mathbf{q}, t)\left|c^{(12)}(\mathbf{q})\right|^{2} .
\end{aligned}
$$

Equations (46), (53), and (55) now lead to

$$
D_{s}^{-1}=\frac{1}{3 \rho_{1}} \int_{0}^{\infty} d t \int d \mathbf{q q}^{2} S_{s}^{(11)}(\mathbf{q}, t) S^{(22)}(\mathbf{q}, t)\left|c^{(12)}(\mathbf{q})\right|^{2},
$$

which can be expressed in terms of measurable parameters of the system once the correlation functions are specified. This is the key result of the present section, and its implications are discussed below. 


\section{DISCUSSION}

\section{A. Scaling relations}

Because the system is close to the critical point of the solvent, it is reasonable to assume that the relevant length scale in the problem corresponds to small $\mathbf{q}$, and that the correlation functions are all adequately represented by their hydrodynamic forms. ${ }^{12}$ For the dynamic structure factors, these $\operatorname{are}^{20}$

$$
\begin{aligned}
S_{s}^{(11)}(\mathbf{q}, t)= & \rho_{1} \mathrm{e}^{-q^{2} D_{s} t}, \\
S^{(22)}(\mathbf{q}, t)= & \rho_{2} \widetilde{S}^{(22)}(\mathbf{q})\left[\left(1-\frac{C_{V}}{C_{P}}\right) \mathrm{e}^{-q^{2} D_{T}}\right. \\
& \left.+\frac{C_{V}}{C_{P}} \cos \left(c_{s} q t\right) \mathrm{e}^{-q^{2} \Gamma t}\right],
\end{aligned}
$$

where $\widetilde{S}^{(22)}(\mathbf{q})$ is the equilibrium structure factor of the solvent, which can be approximated by

$$
\widetilde{S}^{(22)}(\mathbf{q})=\frac{\rho_{2} k_{B} T \kappa_{T} \xi^{-2}}{q^{2}+\xi^{-2}},
$$

with $\kappa_{T}$ the isothermal compressibility and $\xi$ the correlation length of density fluctuations; $C_{V}$ and $C_{P}$ are the constant volume and constant pressure heat capacities, respectively, $D_{T}$ is the thermal diffusivity, $c_{s}$ is the adiabatic speed of sound, and $\Gamma$ is the sound attenuation constant. In order to obtain results for $D_{s}$, that at least initially can be expressed in analytic form, the expression for the dynamic structure factor [Eq. (58)] is further simplified to

$$
S^{(22)}(\mathbf{q}, t) \approx \rho_{2} \widetilde{S}^{(22)}(\mathbf{q}) \mathrm{e}^{-q^{2} D_{T^{t}}},
$$

using the fact that the ratio $C_{V} / C_{P}$ is fairly small near the critcal point. (For ethane, for example, it is between about $10^{-2}$ to $10^{-3}$ in the density range $0.73 \leqslant \rho / \rho_{c} \leqslant 1.16$ along the critical isotherm, ${ }^{21} \rho_{c}$ being the critical density.)

No comparably tractable analytic representation of the direct correlation function $c_{12}(\mathbf{q})$ is known. (One is known for a mixture of hard spheres, ${ }^{22}$ but a hard-sphere model would probably be unsatisfactory near a critical point, since attractive interactions are likely to be important there.) However, $c_{12}(\mathbf{q})$ is known to be finite at the critical point, so it should be possible to approximate it by its long wavelength limit $c_{12}(0)$. Approximations to this quantity are somewhat easier to devise because of the many thermodynamic identities based on fluctuation theory that it is known to satisfy. ${ }^{23}$ For example, in the limit of infinite dilution, it can be shown that $^{24}$

$$
c_{12}(0)=\frac{1}{\rho_{2}}\left[1-\frac{\bar{V}_{1}^{\infty}}{k_{B} T \kappa_{T}}\right],
$$

where $\bar{V}_{1}^{\infty}$ is the partial molar volume of the solute at infinite dilution, an observable property of the system that can (and has) been determined in experiments and simulations. ${ }^{25}$ However, rather than attempt to incorporate such data into our analysis, we simply use the further approximation

$$
c_{12}(0) \approx \frac{1}{\rho_{2}},
$$

which is in the spirit of Mazenko's treatment of the thermal conductivity near the critical point of a one-component liquid. $^{12}$

When Eqs. (57), (60), and (62) are substituted into Eq. (56), the integrals there can be evaluated exactly. (However, a cutoff $\Lambda$ on the integral over wave vectors $\mathbf{q}$ is introduced to ensure finiteness.) $D_{s}$ is then found to be

$$
D_{s}=\frac{D_{T}}{(4 \pi / 9) k_{B} T \kappa_{T} \Lambda^{3} F\left(1,3 / 2 ; 5 / 2 ;-\Lambda^{2} \xi^{2}\right)-1},
$$

where $F(a, b ; c ; z)$ is the Gauss hypergeometric function. ${ }^{26}$ Because the dimensionless distance $\Lambda \xi$ is large, it is useful to rewrite the hypergeometric function in Eq. (63) $\mathrm{as}^{26}$

$$
\begin{aligned}
F\left(1,3 / 2 ; 5 / 2 ;-\Lambda^{2} \xi^{2}\right)= & \frac{3}{\Lambda^{2} \xi^{2}} F\left(1,-1 / 2 ; 1 / 2 ;-\frac{1}{\Lambda^{2} \xi^{2}}\right) \\
& -\frac{3 \pi}{2 \Lambda^{3} \xi^{3}} F\left(3 / 2,0 ; 3 / 2 ;-\frac{1}{\Lambda^{2} \xi^{2}}\right),
\end{aligned}
$$

so that a series expansion can be used to approximate it. When such an approximation is used in Eq. (63), we find

$$
\begin{aligned}
D_{s}^{-1}= & D_{T}^{-1}\left[\frac{4 \pi}{3 \xi^{2}} k_{B} T \kappa_{T} \Lambda\right. \\
& \left.\times\left\{1-\frac{\pi}{2 \Lambda \xi}+\frac{1}{\Lambda^{2} \xi^{2}}-\frac{1}{3 \Lambda^{4} \xi^{4}}+\ldots\right\}-1\right] .
\end{aligned}
$$

From Eq. (65), one sees that $D_{s}$ manifests a scaling form in the reduced temperature $\tau$-near the critical point, ${ }^{27} D_{s}$ varies essentially as $D_{T} \xi^{2} / \kappa_{T}$, but $D_{T} \sim \tau^{0.67}, \xi \sim \tau^{-0.63}$ and $\kappa_{T} \sim \tau^{-1.239}$, and so

$$
D_{s} \sim \tau^{0.65} \text {. }
$$

In terms of the reduced density, $\Delta \rho=\left|\rho-\rho_{c}\right| / \rho_{c}$, the scaling of $D_{s}$ along the critical isotherm is expected to be $\mathrm{be}^{28}$

$$
D_{s} \sim \Delta \rho^{0.65 / \beta}=\Delta \rho^{2.01},
$$

where $\beta=0.324$ is the value of the exponent that characterizes the vanishing of the gas-liquid coexistence curve.

Equation (66) for the scaling of $D_{s}$ with temperature at fixed density is in agreement with predictions based on mode coupling and renormalization group analyses. ${ }^{29}$

\section{B. Numerical estimates of $D_{s}$}

Numerical estimates of $D_{s}$ can be obtained from Eq. (63) if the values of the cut-off $\Lambda$ and the coefficients $D_{T}, \kappa_{T}$, and $\xi$ at different temperatures and densities are specified. $\Lambda$ is the sole parameter related to the solute, and may be identified with an inverse hard-sphere radius. The other parameters relate to the solvent, and for selected solvents, can be obtained from the National Institutes of Science and Technology (NIST) database, ${ }^{21}$ which uses compilations of experimental data on different solvents measured close to and far from the critical region to derive multiparameter equations-of-state for the thermodynamic and transport properties of a given system. In order to illustrate more quantitatively the general trends in the behavior of the diffusion co- 
TABLE I. $D_{s}$ calculated according to Eq. (63) at selected values of the solvent density for a fixed temperature of $307 \mathrm{~K}$.

\begin{tabular}{cc}
\hline \hline $\begin{array}{c}\text { Density } \\
(\mathrm{mol} / \mathrm{L})\end{array}$ & $\begin{array}{c}D_{s} \\
\mathrm{~m}^{2} / \mathrm{s}\left(\times 10^{11}\right)\end{array}$ \\
\hline 5.00 & 22.48 \\
5.25 & 18.33 \\
5.50 & 15.05 \\
5.75 & 12.64 \\
6.00 & 11.04 \\
6.25 & 10.18 \\
6.50 & 9.98 \\
6.75 & 10.40 \\
7.00 & 11.50 \\
7.25 & 13.50 \\
7.50 & 16.80 \\
7.75 & 22.05 \\
8.00 & 30.08 \\
\hline \hline
\end{tabular}

efficient near the critical point, $D_{s}$ is calculated from Eq. (63) as a function of density at a fixed temperature for the solvent ethane assuming that the size of the solute corresponds to a $\Lambda$ arbitrarily assigned the value $1 / 1.9 \mathrm{~A}^{-1}$ based on results for a one-component plasma. ${ }^{13}$ The critical temperature and density of ethane are, respectively, $305.4 \mathrm{~K}$ and $6.87 \mathrm{~mol} \mathrm{~L}^{-1}$, and the NIST database is used to generate its thermodynamic and transport coefficients at a fixed temperature of $307 \mathrm{~K}$ (corresponding to a reduced temperature $\left|1-T / T_{c}\right|$ of $\left.5.24 \times 10^{-3}\right)$ and at a series of densities between $5 \mathrm{~mol} \mathrm{~L}^{-1}$ and $8 \mathrm{~mol} \mathrm{~L}^{-1}$ around the critical density. These values are substituted into Eq. (63) along with the given value of the cutoff to produce $D_{s}$. The results are shown in Table I, where $D_{s}$ is expressed in units of $\mathrm{m}^{2} \mathrm{~s}^{-1}$.

These results have no direct experimental counterparts, but they are of the same order of magnitude as diffusion coefficients measured for a number of different solutesolvent pairs. ${ }^{3}$ Moreover, they are consistent with the observation of near-critical anomalies in the self-diffusion coefficient in the simulations of Drozdov and Tucker, ${ }^{8}$ where the self-diffusion coefficient is first seen to decrease with increase in density at fixed $T$ close to $T_{c}$, then to reach a minumum near $\rho_{c}$, increasing thereafter with further increase in the density, and eventually decreasing again beyond the asymptotic critical region. The simulation results have been ascribed to the existence of local density inhomogeneities around the diffusing species; the results of the present work ultimately derive from a model that neglects all but a single hydrodynamic mode-the solvent density fluctuation-in the dynamical equations governing self-motion. The underlying mechanism of mass transport in both sets of calculations therefore appears to be fundamentally the same.

The estimates of $D_{s}$ in Table I can be refined by improvements on the one hand, to the direct correlation function (along the lines suggested earlier) and on the other, to the self and collective structure factors. For example, an improved description of $S_{s}(\mathbf{q}, t)$ could use $\mathrm{e}^{16}$

$$
S_{s}^{(11)}(\mathbf{q}, t)=\rho_{1} \mathrm{e}^{-q^{2} W(t) / 2},
$$

where

$$
W(t)=2 D_{s}\left[t+\frac{m_{1} D_{s}}{k_{B} T}\left(\mathrm{e}^{-k_{B} T t / m_{1} D_{s}}-1\right)\right] .
$$

This form of the correlation function smoothly interpolates between the long-time limit given by Eq. (57) and the short-time free-particle limit given by $S_{s}^{(11)}(\mathbf{q}, t)=\rho_{1}$ $\times \exp \left[-q^{2} t^{2} k_{B} T / 2 m_{1}\right]$. Similarly, $S^{(22)}(\mathbf{q}, t)$ could be assigned different functional forms in different intervals of the wave vector $q$. In the interval $0 \leqslant q \leqslant 1 / \Lambda$, for example, it could be given by Eq. (58), whereas in the interval $1 / \Lambda \leqslant q$ $\leqslant \infty$, it could be given by ${ }^{30}$

$$
S^{(22)}(\mathbf{q}, t)=\rho_{2} \widetilde{S}^{(22)}(\mathbf{q}) \exp \left(-q^{2} D_{22} t / \widetilde{S}^{(22)}(\mathbf{q})\right),
$$

where $D_{22}$ is the self-diffusion ceofficient of the solvent. These approximations no longer allow $D_{s}$ to be evaluated analytically.

\section{Relation to mode-coupling theory}

Diffusion in binary mixtures is characterized in general by a mutual diffusion coefficient $D$ that contains contributions from both self and cross diffusivities. ${ }^{20}$ Microscopically, $D$ can be expressed as the time integral of an interdiffusion current correlation function, as follows:

$$
D \propto \int_{0}^{\infty} d t\langle\mathbf{J}(t) \cdot \mathbf{J}(0)\rangle,
$$

where

$$
\mathbf{J}(t)=x_{2} \sum_{i=1}^{n_{1}} \mathbf{v}_{i}^{(1)}-x_{1} \sum_{i=1}^{n_{2}} \mathbf{v}_{i}^{(2)} .
$$

Here $x_{\alpha}$ and $n_{\alpha}, \alpha=1,2$ are the mole fraction and number of particles, respectively, of the $\alpha$ th species in the mixture, while $\mathbf{v}_{i}^{(\alpha)}, \alpha=1,2$ is the velocity of the $i$ th particle of this species. The angular brackets refer to an equilibrium ensemble average. In the limit of vanishing amounts of one component, say component 2 , corresponding to the solute, $D$ reduces to the self-diffusion coefficient $D_{0}$ of that component, and is expressible therefore as the time integral of a velocity-velocity time correlation function for a tagged solute particle in an excess of the solvent. In general, $D_{0}$ is the sum of a background part $D_{b}$ that is an analytic function of its thermodynamic arguments, and an excess part $\Delta D$ that is nonanalytic and that dominates the behavior of $D_{0}$ in the near vicinity of the critical point. $\Delta D$ has been found from mode-coupling theory ${ }^{31}$ and other variants of the theory ${ }^{32,33}$ to have the general form

$$
\Delta D=\frac{k_{B} T}{6 \pi \eta \xi} \Omega\left(q_{c} \xi\right),
$$

where $\eta$ and $\xi$ are the solvent viscosity and correlation length, respectively, $q_{c}$ is a cutoff on the wave vector $\mathbf{q}$, and $\Omega\left(q_{c} \xi\right)$ is a so-called crossover function that interpolates between the asymptotic critical regime where $q_{c} \xi \rightarrow \infty$ and the noncritical regime where $q_{c} \xi \rightarrow 0$.

Near the critical point, the thermal diffusivity is in general given by ${ }^{27} k_{B} T / 6 \pi \eta \xi$, so Eq. (63) for $D_{s}$ in the present calculations actually manifests the same functional form as $\Delta D$ above if the function $1 /\left[(4 \pi / 9) k_{B} T \kappa_{T} \Lambda^{3} F(1,3 / 2 ; 5 / 2\right.$; 
$\left.\left.-\Lambda^{2} \xi^{2}\right)-1\right]$ is identified with the crossover function $\Omega\left(q_{c} \xi\right)$, the cut-off $q_{c}$ being equated with $\Lambda$. By appropriate adjustment of $\Lambda$, the crossover function $\Omega\left(q_{c} \xi\right)$ so identified can be made to approach unity in the limit $\Lambda \xi \rightarrow \infty$, thereby producing a Stokes-Einstein form for the diffusion coefficient.

The more general formula for $D_{s}$ is given by Eq. (56). The right-hand side of this equation is actually identical (up to numerical constants) to the expression for the memory function associated with density fluctuations in dense or supercooled liquids derived on the basis of a density functional formulation of mode-coupling theory. ${ }^{34}$ The memory function in such theories is in general the time correlation function of a random force, and is related to the friction coefficient of the solute, which in turn is related to its diffusion coefficient by the Stokes-Einstein equation. The random force may be identified with an osmotic force originating in the excess chemical potential due to interparticle interactions. ${ }^{35}$ The excess chemical potential in turn is the real-space convolution of a direct pair correlation function and the deviation of the collective number density from its mean value. The product of the local density of the tagged particle and the gradient of the chemical potential is directly proportional to the force, and the force-force correlation function obtained thereby can be shown, in Fourier space, to have the same structure as the right-hand side of Eq. (56).

Thus, the present kinetic theory approach within the disconnected approximation and applicable near the critical point of the solvent is effectively equivalent to mode coupling theories of binary mixtures of slow dense liquids.

${ }^{1}$ P. C. Hohenberg and B. I. Halperin, Rev. Mod. Phys. 49, 435 (1977); N. Goldenfeld, Lectures on Phase Transitions and the Renormalization Group (Addison-Wesley, Reading, MA, 1992); J. V. Sengers and J. Luettmer Strathmann, in Transport Properties of Fluids: Their Correlation, Prediction and Estimation, edited by J. Millat, J. H. Dymond, and C. A. Nieto de Castro (Cambridge University Press, New York, 1996), p. 113.

${ }^{2}$ R. Zwanzig, J. Chem. Phys. 34, 1931 (1961); B. Otto, J. Schroeder, and J. Troe, J. Chem. Phys. 81, 202 (1984); G. Maneke, J. Schroeder, J. Troe, and F. Voss, Ber. Bunsenges. Phys. Chem. 89, 896 (1985); D. Schwarzer, J. Troe, M. Votsmeier, and M. Zerezke, J. Chem. Phys. 105, 3121 (1996); J. Benzler, S. Linkersdorfer, and K. Luther, ibid. 106, 4992 (1997).

${ }^{3}$ N. J. Trappeniers and P. H. Oosting, Phys. Lett. 23, 445 (1966); V. G. Martynets and E. V. Matizen, Sov. Phys. JETP 31, 228 (1970); ibid. 40, 507 (1975); Yu. V. Tsekhanskaya, Russ. J. Phys. Chem. 45, 744 (1971); W. J. Lamb, G. A. Hoffman, and J. Jonas, J. Chem. Phys. 74, 6875 (1981); C. K. J. Sun and S.-H. Chen, AIChE J. 31, 1510 (1985); Chem. Eng. Sci. 40, 2217 (1985); D. M. Lamb, S. T. Adamy, K. W. Woo, and J. Jonas, J. Phys. Chem. 93, 5002 (1989); A. A. Clifford and S. E. Coleby, Proc. R. Soc. London, Ser. A 433, 63 (1991); K. K. Liong, P. A. Wells, and N. R. Foster, J. Supercrit. Fluids 4, 91 (1991); S. Umezawa and A. Nagashima, ibid. 5, 242 (1992); V. M. Shenai, B. L. Hamilton, and M. A. Mathews, in Supercritical Fluid Engineering Science, edited by E. Kiran and J. F. Brennecke
(American Chemical Society, Washington, D.C., 1993); A. Akgerman, C. Erkey, and M. Orejuela, Ind. Eng. Chem. Res. 35, 911 (1996); H. Nishiumi, M. Fujita, and K. Agon, Fluid Phase Equilib. 117, 356 (1996); S. Tsuzuki, T. Uchimaru, M. Mikami, K. Tanabe, T. Sako, and S. Kuwajima, Chem. Phys. Lett. 225, 347 (1996); Y. Kimura, D. Kanda, M. Terazima, and N. Hirota, J. Phys. Chem. B 101, 4442 (1997).

${ }^{4}$ S.-H. Chen, Chem. Eng. Sci. 38, 655 (1983); H. Liu and E. A. Macedo, J. Supercrit. Fluids 8, 310 (1995); H. Liu and E. Ruckenstein, Ind. Eng. Chem. Res. 36, 888 (1997); A. P. Eaton and A. Akgerman, ibid. 36, 923 (1997); H. Liu, C. M. Silva, and E. A. Macedo, ibid. 36, 246 (1997).

${ }^{5}$ T. Keyes, J. Chem. Phys. 62, 1691 (1975).

${ }^{6}$ M. Gitterman, Phys. Rev. E 52, 303 (1995).

${ }^{7}$ E. P. Sakonidon, H. R. van den Berg, C. A. ten Seldam, and J. V. Sengers, J. Chem. Phys. 109, 717 (1998); E. V. Matizen, P. P. Bezverkhy, and V. G. Martynets, Phys. Rev. E 59, 2927 (1999).

${ }^{8}$ A. N. Drozdov and S. Tucker, J. Chem. Phys. 114, 4912 (2001).

${ }^{9}$ S. Bhattacharya and B. Bagchi, J. Chem. Phys. 106, 1757 (1997).

${ }^{10}$ G. F. Mazenko, Phys. Rev. A 7, 209 (1973).

${ }^{11}$ G. F. Mazenko, Phys. Rev. A 7, 222 (1973).

${ }^{12}$ G. F. Mazenko, Phys. Rev. A 9, 360 (1974).

${ }^{13}$ J. I. Castresana, G. F. Mazenko, and S. Yip, Ann. Phys. (N.Y.) 103, 1 (1977); Phys. Rev. A 14, 1814 (1976).

${ }^{14}$ H. Gould and G. F. Mazenko, Phys. Rev. Lett. 35, 1455 (1975).

${ }^{15}$ G. F. Mazenko and S. Yip, in Modern Theoretical Chemistry, edited by B. J. Berne (Plenum, New York, 1977), Vol. 6, p. 181.

${ }^{16}$ S. Yip, Annu. Rev. Phys. Chem. 30, 547 (1979).

${ }^{17}$ G. F. Mazenko and S. Yip, in Molecular Motions in Liquids, edited by J. Lascombe (Reidel-Dodrecht, Holland, 1974), p. 79.

${ }^{18}$ T. Yamaguchi, N. Matubayasi, and M. Nakahara, J. Chem. Phys. 115, 422 (2001).

${ }^{19}$ J. R. Mehaffey and R. I. Cukier, Phys. Rev. A 17, 1181 (1978).

${ }^{20}$ J. P. Hansen and I. R. McDonald, Theory of Simple Liquids (Academic, New York, 1990).

${ }^{21}$ D. G. Friend, NIST Mixture Property Database Version 9.08 (U.S. Department of Commerce, 1992.)

${ }^{22}$ J. L. Lebowitz, Phys. Rev. A 133, 895 (1964).

${ }^{23}$ J. G. Kirkwood and F. P. Buff, J. Chem. Phys. 19, 774 (1951)

${ }^{24}$ I. B. Petsche and P. G. Debenedetti, J. Phys. Chem. 95, 386 (1991).

${ }^{25}$ C. A. Eckert, D. H. Ziger, K. P. Johnston, and S. Kim, J. Phys. Chem. 90, 2738 (1986); R. F. Chang and J. M. H. Levelt Sengers, J. Phys. Chem. 90, 5921 (1986).

${ }^{26}$ I. S. Gradshteyn and I. M. Ryzhik, Tables of Integrals, Series and Products (Academic, Boston, 1994).

${ }^{27}$ J. Luettmer-Strathmann, J. V. Sengers, and G. A. Olchowy, J. Chem. Phys. 103, 7482 (1995).

${ }^{28}$ J. V. Sengers and P. H. Keyes, Phys. Rev. Lett. 26, 70 (1971).

${ }^{29}$ E. E. Gorodetskii and M. S. Giterman, Sov. Phys. JETP 30, 348 (1970); L. Mistura, Nuovo Cimento Soc. Ital. Fis., B 12B, 35 (1972); J. Chem. Phys. 62, 4571 (1975); A. Onuki, J. Low Temp. Phys. 61, 101 (1985); M. A. Anisimov and S. B. Kiselev, Int. J. Thermophys. 13, 873 (1992); R. Folk and G. Moser, J. Low Temp. Phys. 99, 11 (1995); Int. J. Thermophys. 75, 2706 (1995); Phys. Rev. Lett. 16, 1363 (1995).

${ }^{30}$ M. Medina-Noyola, Phys. Rev. Lett. 60, 2705 (1988).

${ }^{31}$ J. Luettmer-Strathmann and J. V. Sengers, J. Chem. Phys. 104, 3026 (1996).

${ }^{32}$ S. B. Kiselev and V. D. Kulikov, Int. J. Thermophys. 15, 283 (1994); 18, 1143 (1997)

${ }^{33}$ S. B. Kiselev and M. L. Huber, Fluid Phase Equilib. 142, 253 (1998).

${ }^{34}$ S. M. Ali, A. Samanta, and S. K. Ghosh, J. Chem. Phys. 114, 10419 (2001).

${ }^{35}$ A. V. Indrani and S. Ramaswamy, Phys. Rev. Lett. 73, 360 (1994). 